\title{
Faire évoluer les systèmes adultes vers des pratiques restauratives dans une middle-school américaine
}

Moving adult systems towards restorative practices in an American middle school

Hacer evolucionar los sistemas adultos hacia unas prácticas restauradoras en una middle-school norteamericana

Anne Gregory et Suzanne Hitchman

Traducteur : Sylvaine Herold

\section{OpenEdition} Journals

Édition électronique

URL : https://journals.openedition.org/ries/8802

DOI : 10.4000/ries.8802

ISSN : 2261-4265

Éditeur

France Education international

Édition imprimée

Date de publication : 1 septembre 2019

Pagination : 97-107

ISBN : 978-2-85420-624-1

ISSN : 1254-4590

Référence électronique

Anne Gregory et Suzanne Hitchman, «Faire évoluer les systèmes adultes vers des pratiques restauratives dans une middle-school américaine », Revue internationale d'éducation de Sèvres [En ligne], 81 | septembre 2019, mis en ligne le 01 septembre 2021, consulté le 14 octobre 2021. URL : http:// journals.openedition.org/ries/8802 ; DOI : https://doi.org/10.4000/ries.8802 


\title{
Faire évoluer les systèmes adultes vers des pratiques restauratives dans une middle-school américaine $^{*}$
}

\author{
Anne Gregory \\ Université Rutgers \\ Suzanne Hitchman \\ Consultante
}

Aux États-Unis, les professionnels du secteur éducatif ont de plus en plus recours à la justice restaurative ou aux pratiques restauratives dans les établissements scolaires. Ces efforts s'inscrivent dans le cadre d'un mouvement croissant en faveur d'un moindre recours aux approches punitives face à l'inconduite des élèves - un enjeu particulièrement important vis-à-vis des élèves de couleur. Des données de plus en plus nombreuses montrent en effet que les élèves noirs ont tendance à recevoir des sanctions plus sévères que les élèves blancs pour un comportement similaire (voir par exemple Owens et McLanahan, 2018). Les pratiques restauratives (PR) sont une approche fondée sur quelques grands principes pour le développement de communautés et la résolution des conflits. Il ne s'agit pas de programmes d'action statiques mis en œuvre de manière étroite ou standardisée dans les établissements. Mais les véritables défis de la mise en œuvre des PR dans des établissements sous-financés, soumis à la pression d'un environnement d'examens à enjeux élevés, ont été peu étudiés. La présente étude de cas apporte un éclairage sur le renforcement des capacités des systèmes adultes pour mener à bien un projet de PR dans un contexte d'enjeux concrets. Renforcer les capacités signifie à la fois agir sur l'évolution des convictions, des mentalités et renforcer les politiques, les systèmes et les structures nécessaires pour soutenir les PR.

\section{DÉFINIR LES PRATIQUES RESTAURATIVES}

Les pratiques restauratives (PR) sont issues des traditions autochtones de résolution de problèmes fondées sur la communauté et de la justice restaurative dans le système juridique. Dans la sphère éducative, elles requièrent un état d'esprit centré sur les relations, de telle sorte que la pratique consistant à renforcer et rétablir les relations imprègne les interactions quotidiennes des membres du personnel et

\footnotetext{
* Article traduit par Sylvaine Herold.
} 
des élèves et soit profondément ancrée dans le fonctionnement des établissements. Thorborne, Riestenberg et McCluskey (2019) affirment que les PR sont, fondamentalement, " des pratiques et des interventions de mise en relation, qui cherchent à rétablir et à renforcer les relations » (p. 16). Ainsi, les PR s'inscrivent dans un continuum prévention-intervention. C'est-à-dire que certaines pratiques préviennent les infractions par l'élaboration d'une communauté tandis que d'autres interviennent après les infractions (voir par exemple Amstutz et Mullet, 2005).

Les PR axées sur la prévention sont : (a) les énoncés affectifs utilisés par les adultes et les élèves pour exprimer leurs émotions, lorsque des conflits surgissent (par exemple, " je me sens frustré lorsque vous faites cela ») ; (b) les processus équitables, en tant que principe pour faire en sorte que les administrateurs ou les enseignants prennent véritablement en compte le point de vue des personnes concernées par les décisions (Wachtel, 2016) ; et (c) les pratiques de renforcement de la communauté telles que l'organisation de cercles hebdomadaires ou mensuels avec les membres du personnel et les élèves. Boyes-Watson et Pranis (2014) décrivent les cercles comme un processus de communication structuré permettant d'accroître les compétences sociales et émotionnelles des participants. Les participants développent des compétences de partage sur eux-mêmes et d'écoute des autres. Ils sont assis en cercle et se font face, symbolisant un pouvoir conjoint et une hiérarchie réduite. Pour assurer une pratique inclusive, chaque participant a la possibilité de parler quand on lui passe un «bâton de parole ».

Les PR axées sur l'intervention, visant à résoudre les conflits (ou les infractions aux règles), comprennent : (a) les cercles de résolution de problèmes, la médiation et les conversations informelles restauratives ; (b) les cercles de réintégration visant à réintégrer et à soutenir les élèves revenant d'absences prolongées (par exemple, une exclusion de longue durée) ; (c) les conférences de restauration formelles visant à réparer les torts lorsque surviennent des dommages " plus graves » (Berkowitz, 2019). Lors de ces conférences, toutes les personnes impliquées dans un incident ayant causé un préjudice sont invitées à participer. Toutes s'assoient en cercle et se font face, et un facilitateur utilise un ensemble structuré de questions pour guider le processus. Ainsi, l'ensemble des personnes impliquées a la possibilité de réfléchir et de répondre à des questions telles que : «Que s'est-il passé ? »; «Qui a été blessé/affecté par ce que vous avez fait ?»; «Quelle est votre part de responsabilité ?»; "Comment les torts pourront-t-ils être réparés ?» (Wachtel et al., 2009). La conférence peut aboutir à la définition d'un plan de réparation du préjudice.

L'approche en termes de PR dans les établissements scolaires est considérée comme un changement de paradigme, à l'opposé de l'approche punitive de l'inconduite des élèves. Wachtel (2016) souligne que travailler dans une optique restaurative est une manière d' " être » et de "faire ", par laquelle les adultes et les élèves travaillent ensemble, à l'opposé d'une approche où les adultes agiraient "sur » les élèves de manière autoritaire.

D'autres théoriciens ont également souligné qu'un autre principe fondateur des PR est la création d'environnements d'apprentissage justes et équitables (Evans et Vaandering, 2016). D'autres encore, se fondant sur la théorie de l'attachement, soulignent que des communautés d'appartenance et de confiance inclusives sont une condition préalable à l'apprentissage et au développement (Sabol et Pianta, 2012). 


\section{L'ÉTUDE DE CAS : \\ UNE MIDDLE-SCHOOL ${ }^{1}$ AMÉRICAINE}

Dans notre étude, un important district urbain a sélectionné quelques établissements pour un partenariat avec une fondation locale qui a apporté le financement pour un projet de PR d'une durée de quatre ans. Nous proposons d'étudier ici le cas de l'un de ces établissements, où les fonds ont permis à une directrice de site $\mathrm{PR}$, issue d'une organisation à but non lucratif, d'occuper un poste à temps plein au sein de l'établissement (Hitchman, la deuxième auteure). Les fonds ont également permis l'évaluation du projet par une université locale (Gregory, la première auteure). Pour cette étude de cas, nous nous appuyons sur des données provenant de différentes sources: (a) entretiens annuels avec la directrice de site PR, enregistrements et notes de terrain en tant qu'observatrice participante ; (b) entretiens avec les élèves; et (c) enquêtes auprès des élèves et du personnel à partir de la deuxième et troisième années de mise en œuvre.

Létablissement partage un grand bâtiment avec deux autres écoles. En se rendant à son étage supérieur, le visiteur découvre des peintures murales colorées, sur lesquelles on peut lire "None but ourselves can free our minds $»^{2}$ au-dessus d'une représentation de Bob Marley ou encore "Beat the drums " ${ }^{3}$ aux côtés de héros et d'activistes locaux, Jack Robinson ${ }^{4}$, Shirley Chisolm ${ }^{5}$ et Colin Kaepernick ${ }^{6}$. Les couloirs sont décorés de photos d'élèves et de leurs familles prises lors d'événements récents, d'exemples de travaux d'élèves et d'une affiche présentant les élèves désignés « meilleurs joueurs $»^{7}, c^{\prime}$ est-à-dire ceux contribuant de manière positive à la communauté aux niveaux scolaire et social.

L'établissement accueille près de 150 élèves, des grades 6 à $8^{8}$. Les élèves inscrits sont à $63 \%$ noirs, $28 \%$ latinos, $4 \%$ asiatiques, $3 \%$ autres et $2 \%$ blancs. Les membres du personnel sont en majorité des femmes et des personnes de couleur. Les élèves et les membres du personnel sont issus de milieux culturels et ethniques variés. De nombreuses familles du quartier ont émigré des Caraïbes - certaines sont arrivées en masse dans les années 1960, d'autres plus récemment. L'établissement se trouve d'ailleurs à proximité du point de départ du défilé annuel de la Journée des Caraïbes?, qui célèbre le patrimoine culturel des résidents trinidadiens, jamaïcains, barbadiens, haïtiens, saint-luciens et guyanais. Une part importante des élèves (19\%) sont des

1. Ce qui correspond à un établissement d'enseignement secondaire inférieur. (NdT)

2. Nul autre que vous ne peut libérer votre esprit. (NdT)

3. Battez les tambours. (NdT)

4. Jack Roosevelt Robinson, dit Jackie Robinson (1919-1972), est un joueur américain de baseball. Militant infatigable du combat pour les droits civiques, il est le premier Noir à jouer en Ligue majeure en 1947. (NdT)

5. Shirley Chisholm (1924-2005) est la première femme afro-américaine élue au Congrès américain en 1968. Elle y siège durant sept mandats, de 1969 à 1983. En 1972, elle se porte candidate à l'investiture démocrate pour l'élection présidentielle : elle obtient 152 voix mais est largement battue par George McGovern. (NdT)

6. Colin Rand Kaepernick est un joueur de football américain devenu le centre de l'attention médiatique lors de la saison 2016, lorsqu'il s'agenouille pendant l'hymne national américain afin de protester contre le racisme aux États-Unis et les violences policières envers les minorités. $(\mathrm{NdT})$

7. Most valuable players en anglais. (NdT)

8. Soit de 11 à 14 ans. (NdT)

9. La West Indian Day Parade est l'évènement principal du West Indian Carnival (ou Labor Day Parade), une célébration annuelle qui a lieu le jour de la fête du travail américaine (le premier lundi de septembre) à Crown Heights, Brooklyn, New York. (NdT) 
apprenants de l'anglais, dont beaucoup sont arrivés récemment de pays comme la Guinée ou le Yémen. Du fait du faible niveau de revenu des ménages dont sont issus les élèves, $96 \%$ d'entre eux ont le droit à un déjeuner gratuit ou à prix réduit. En outre, différents facteurs de stress, liés au racisme, à des traumatismes et à une pauvreté structurelle, font que les élèves arrivent à l'école avec de nombreux besoins en termes éducatifs et psychosociaux.

Le projet de PR a été initié au beau milieu de la réforme de la discipline scolaire à l'échelle du district. Le code de discipline du district avait déjà été révisé afin de réduire le temps d'enseignement perdu en raison des exclusions. Le code révisé encourage les professionnels du secteur éducatif à intervenir via des dispositifs de soutien comportemental en cas de violation des règles. Signalons également que le code révisé exige désormais des chefs d'établissement qu'ils obtiennent l'approbation écrite du district pour pouvoir prononcer une exclusion hors de l'école de 1 à 5 jours pour " provocation » ou " désobéissance », contrairement à ce qui se faisait auparavant. Ce changement de politique a probablement contribué à la réduction globale du nombre d'exclusions à l'échelle du district.

Pour bien saisir le contexte de cet établissement, il est également important de noter qu'il s'agit d'un établissement placé sous la surveillance étroite de l'administration du district. Au début du projet, en effet, les élèves avaient obtenu des résultats sensiblement inférieurs à la moyenne du district. Dès lors, le personnel a collectivement subi des pressions afin de consacrer le plus de temps possible à l'enseignement de contenu académique. Au cours des quatre années du projet, leurs efforts ont porté leurs fruits et les résultats des élèves se sont améliorés.

\section{LA MISE EN GUVRE DES PR DANS CET ÉTABLISSEMENT}

La directrice PR du site et ses collègues au sein de l'établissement fixent chaque année des objectifs en fonction d'indicateurs de mise en ouvre des PR (Gregory, 2018). Les objectifs concernaient les indicateurs suivants : a) renforcer l'infrastructure pour les PR (par exemple, harmoniser les politiques en matière de PR et de discipline) ; b) proposer un développement professionnel formel et informel aux enseignants et aux administrateurs (formations, co-facilitation de cercles, par exemple) ; c) construire une communauté (cercles, événements) ; d) réparer les dommages (conférences, coaching en gestion de conflits) ; e) favoriser le leadership des jeunes ; f) adopter des pratiques explicites en termes de/d'(in)justice raciale et sociale ; et g) impliquer les familles aux PR.

Plus précisément, les efforts de renforcement de la communauté ont compris les activités suivantes : la conduite de cercles avec les élèves par le personnel enseignant lors des «temps de conseil $»^{10}$ mensuels ou bien pendant les heures de

10. Les advisory periods ou advisories sont des temps dédiés réguliers organisés entre un enseignant et un petit groupe d'élèves, dans le but de les conseiller sur des questions scolaires, sociales ou relatives à leur avenir. L'objectif est de s'assurer que chaque élève est connu d'au moins un adulte de l'établissement afin de veiller à ce que ses besoins d'apprentissage soient satisfaits et de l'encourager à penser à son avenir. D'une durée approximative de 20-30 minutes, ces temps de conseil sont conçus pour renforcer les relations adultes-élèves ainsi que le sentiment d'appartenance et de communauté des élèves. (Source : The Glossary of Education Reform, https:// www.edglossary.org/advisory/). (NdT) 
classe, à la discrétion des enseignants. Les cercles avec l'ensemble de la classe rassemblaient 18 à 25 élèves environ pendant 15 à 45 minutes. En cercle, les élèves ont pu échanger sur des sujets tels que la pertinence du matériel pédagogique d'un point de vue personnel, leurs inquiétudes quant aux prochains examens d'importance à venir ou la manière de résoudre les conflits en classe.

En cas de difficultés interpersonnelles ou de préjudice, le personnel d'appui (assistants éducatifs, mentors, spécialistes du comportement) pouvait conduire des cercles plus restreints, de 2 à 10 élèves. La durée de ces cercles pouvait varier de 30 à 120 minutes en fonction de la complexité du problème et de la disposition des participants à s'exprimer avec sincérité. Ces cercles pouvaient être initiés par des membres du personnel ou bien par des élèves afin de réparer les torts causés. En fait, il est devenu courant de voir des élèves se rapprocher d'un membre du personnel de confiance en déclarant : « Nous avons besoin d'un cercle!».

\section{RENFORCER L'INFRASTRUCTURE ET LES CAPACITÉS ADULTES EN MATIÈRE DE PR}

Au début du projet, malgré la formulation d'objectifs globaux, il est devenu évident que la mise en ouvre rapide d'activités telles que les cercles et les conférences pouvait s'avérer risquée sans renforcement préalable des capacités des adultes. Notamment, en l'absence de capacités adultes appropriées et de procédures claires, on craignait que le personnel de l'établissement puisse, par exemple, expérimenter les cercles avec leurs élèves uniquement pour s'efforcer de maintenir l'engagement et la coopération des élèves. Ce qui pourrait conduire à la conclusion prématurée que les PR sont un échec; ou bien aboutir à ce que les membres du personnel ne transforment un processus restaurateur en un processus punitif, favorisant ainsi la méfiance des élèves. C'est pourquoi le groupe de travail sur les PR a axé ses efforts sur l'adhésion du personnel et le renforcement des capacités, en se focalisant sur le bien-être du personnel, sur le développement et la restauration des relations, en veillant à ce que les PR ne soient pas cloisonnées et en élaborant des systèmes et des structures fonctionnels afin de soutenir le bien-être social et émotionnel des élèves et la santé globale de l'organisation.

\section{Une attention particulière portée au bien-être du personnel et au développement des relations}

Au cours de la première année, la mise en œuvre du projet a suivi le postulat selon lequel les adultes eux-mêmes avaient besoin d'une forte capacité à développer des relations les uns avec les autres et de bien-être émotionnel pour pouvoir appliquer les PR aux élèves. Comme cela a été mentionné précédemment, le personnel était soumis à une très forte pression pour améliorer les résultats des élèves aux examens, et les tensions pouvaient être importantes. Précédemment, peu d'efforts 
avaient été faits pour prendre en compte le stress et le sentiment de communauté du personnel. L'établissement a alors commencé à organiser des mercredis du mieuxêtre à l'heure du déjeuner, pendant lesquels les membres du personnel pouvaient à la fois se nourrir sainement, participer ensemble à des cercles et apporter un soutien à leurs pairs. Ces cercles de 45 minutes menés par les membres du personnel ont porté sur des sujets variés, comprenant la gestion du stress, l'expression par des pratiques artistiques, ou des consultations sur les défis professionnels.

Les cercles de développement communautaire entre adultes ont en outre été intégrés à différentes instances tout au long du projet. Une équipe s'est ainsi mise à utiliser les cercles pour impliquer les parents lors des forums annuels, afin d'obtenir des retours critiques sur les points forts de l'établissement et les stratégies nécessaires pour créer un environnement accueillant et ouvert pour les familles. Les cercles d'adultes ont également été utilisés lors de séances de développement professionnel, de réunions organisées par le représentant syndical pour discuter des relations entre le personnel et les administrateurs, et pour résoudre les problèmes scolaires, comportementaux et d'assiduité d'élèves en particulier. Les membres du personnel ont ainsi pu apprendre à faciliter des cercles avec les élèves en voyant leurs collègues en faire la démonstration. La capacité de réflexion et la vulnérabilité émotionnelle des membres du personnel se sont ainsi accrues. À la fin de la deuxième année du projet, le personnel était en mesure de réfléchir de manière ouverte aux suggestions des élèves sur la manière de rendre l'école plus restaurative. Les cercles ont également renforcé le sentiment d'appartenance communautaire, ainsi que la cohésion, l'unité et la résilience des équipes. Grâce à ce surplus d'unité, la directrice PR a observé une collaboration accrue du personnel et une meilleure communication pour répondre aux besoins des élèves.

Le développement professionnel en matière de $\mathrm{PR}$ était un autre aspect crucial du renforcement des capacités. À la fin de la deuxième année du projet, plus de $80 \%$ du personnel de l'établissement, y compris les enseignants, les assistants éducatifs et les administrateurs, avaient participé à au moins l'une des activités suivantes : (a) une formation aux PR d'une à deux heures, (b) une formation au dialogue restaurateur, ou (c) une formation aux cercles de cinq jours, proposée par le district. En outre, le personnel enseignant et les administrateurs ont bénéficié d'une communauté d'apprentissage professionnel, en groupe restreint, au cours des troisième et quatrième années du projet, ce qui leur a permis d'approfondir le sens d'un état d'esprit restaurateur.

\section{Décloisonner les PR}

Trop souvent, le travail de restauration se limite à des discussions et à des conférences restauratives lorsque les élèves ont enfreint une règle. Le personnel en charge des PR cherche alors à "éteindre l'incendie » et les enseignants envoient les élèves à l'équipe $\mathrm{PR}$ " pour restauration ». Dès lors, le travail de restauration se trouve réduit à néant et les $\mathrm{PR}$ sont identifiées comme relevant de la compétence de quelques membres du personnel seulement. Dans notre établissement, il a été reconnu que les personnes en charge des $\mathrm{PR}$ ne pouvaient être les seules à répondre au comportement des élèves par une approche de résolution de problèmes (discussions restauratives). Des infrastructures devaient être développées pour répondre 
collectivement à l'inconduite des élèves et aux besoins sous-jacents. Le développement des relations et la réparation des dommages devaient être une responsabilité partagée.

L'engagement collectif s'est ainsi développé au fil du projet. Au cours de la deuxième année du projet, seuls huit répondants à l'enquête auprès du corps enseignant ont déclaré avoir facilité un ou plusieurs cercles de développement communautaire. Ils étaient au nombre de 19 au cours de la troisième année du projet. Les équipes ont commencé à assumer personnellement la responsabilité de développer les relations et de réparer les dommages en ayant recours à des approches restauratives en classe, comme cela est illustré par les trois exemples qui suivent. Premièrement, un professeur d'arts a eu recours à un dialogue restaurateur avec des élèves, après que ceux-ci eurent causé une perturbation majeure pendant un cours animé par un artiste-enseignant. Le professeur d'arts a demandé à ses élèves : "Que faudrait-il faire pour corriger cela ? » Et les élèves ont proposé : « Nous devrions organiser un cercle en classe pour parler de ce qui s'est passé et assumer la responsabilité de nos actes ». Ce cercle s'est tenu peu de temps après. Deuxièmement, un enseignant en éducation spécialisée a pris l'initiative d'organiser un cercle sur la sécurité dans la rue après que deux étudiants ont été approchés par des inconnus sur le chemin de l'école. Troisièmement, un autre enseignant s'est associé à un para-professionnel pour conduire un cercle sur les tactiques de désescalade et les stratégies préventives au début du printemps, lorsque l'énergie des élèves et les conflits entre pairs sont décuplés. Ces changements organiques dans les pratiques ont été le résultat de la confluence de la formation continue, du coaching, du soutien administratif, d'une pression collégiale positive entre pairs, ainsi que d'attentes et d'encouragements partagés.

\section{Élaborer des systèmes et des structures en faveur du bien-être social et émotionnel des élèves}

Il a également fallu créer, collaborativement au sein des équipes, des systèmes et des structures pour bâtir l'infrastructure et la pratique d'une approche centrée sur les relations. Grâce à son rôle au sein de l'équipe de direction pédagogique et d'autres équipes scolaires, la directrice PR a pu encadrer les responsables de l'établissement afin qu'ils intègrent les approches relationnelles à leurs interventions. Ainsi, l'équipe en charge des questions d'assiduité a commencé à utiliser un modèle de mentor de classe afin de suivre et de favoriser les progrès de l'assiduité. Plus précisément, un mentor a été associé à chaque classe afin de développer les relations avec les élèves et les familles en ayant recours à une approche holistique de la résolution de problèmes pour expliquer les raisons de l'absentéisme des élèves. De la même façon, la directrice PR a collaboré avec les administrateurs pour restructurer l'équipe de soutien à la réussite des élèves (Student Success Support Team, SSST). Cette équipe a mis au point une approche centrée sur le potentiel et les relations pour gérer le cas des élèves aux niveaux 2 et 3 - ceux ayant besoin de davantage de soutien comportemental et scolaire. Les membres du personnel ont été chargés d'explorer, de conce- 
voir des solutions, de développer des relations et de faire des recommandations en faveur d'interventions proactives non punitives.

Une politique de discipline progressive a également été mise au point au sein de l'établissement et deux spécialistes du comportement ont été chargés de mettre en œuvre des approches restauratives (dialogues restaurateurs, feuilles de réflexion, médiations, par exemple). La politique de discipline progressive définit le rôle des enseignants, des spécialistes du comportement, du personnel de soutien et des administrateurs dans le traitement des infractions comportementales, tout en indiquant clairement les étapes à suivre en fonction de la gravité des infractions. Un formulaire d'aiguillage, adapté du district scolaire unifié de San Francisco, a été introduit pour encourager la réflexion des enseignants et documenter la nature des infractions et des causes profondes présumées avant d'en référer pour action disciplinaire ou enquête. L'objectif est de donner aux enseignants la possibilité de réfléchir au comportement des élèves, d'évaluer leurs besoins et de décider si une intervention restaurative mise en ouvre au niveau de l'enseignant est appropriée. Mais il fournit également aux enseignants des procédures claires pour bénéficier de l'aide d'un spécialiste du comportement lorsqu'une intervention au niveau de l'enseignant n'est pas suffisante - par exemple, en cas de crise, de bagarre, de possession d'armes ou de dommages matériels importants. Les élèves orientés deux fois ou plus vers un spécialiste du comportement sont automatiquement dirigés vers l'équipe SSST afin de bénéficier d'une prise en charge plus intensive et continue de la part de l'équipe de soutien.

\section{Vers un climat scolaire plus restaurateur}

L'établissement a accompli des progrès importants dans la mise en place d'un climat scolaire restaurateur, au sein duquel les élèves et les membres du personnel ont pu développer, en conscience, des relations positives et procéder à la réparation des dommages en cas de conflits. Il convient de noter que, les trois années précédant le projet, 136 exclusions hors de l'école avaient été prononcées dans cet établissement. En revanche, au cours des trois premières années du projet, on a estimé à 35 le nombre d'exclusions ${ }^{11}$. Cela représente une réduction de $74 \% \mathrm{du}$ nombre d'exclusions. Les enquêtes et entretiens menés auprès des élèves corroborent ces résultats positifs.

Nous avons mené une enquête auprès des élèves sur le climat et la discipline dans l'établissement. Au printemps 2017, nous avons collecté 146 questionnaires (soit un taux de réponse de $82 \%$ ). Nous avons notamment cherché à savoir si les groupes d'élèves traditionnellement les plus soumis aux mesures disciplinaires (les élèves noirs, les élèves à besoins éducatifs particuliers, les élèves lesbiens, gays, bisexuels et homosexuels) avaient une expérience de l'école similaire ou différente de celle de leurs pairs. Il est encourageant de constater que, par rapport à leurs pairs, les élèves noirs, les élèves à besoins éducatifs particuliers et les élèves issus de minorités sexuelles ressentaient des niveaux similaires de soutien positif de la part des adultes.

Dans un sous-échantillon d'élèves pour lequel nous disposons de données

11. Le nombre d'exclusions est estimé pendant la période du projet de PR, étant donné que les données annuelles du district sont épurées lorsque moins de cinq élèves se voient imposer une exclusion à court ou long terme. 
complètes, des analyses statistiques ont montré qu'être un élève noir dans cet établissement n'était pas associé à plus de mesures d'exclusion autodéclarées (être invité à quitter la salle de classe pour des raisons comportementales, être exclu ; Gaines, 2018). Ce résultat est notable car il va à l'encontre de la surreprésentation bien documentée des élèves noirs dans les mesures disciplinaires dans tous les établissements scolaires américains (voir par exemple Owens et McLanahan, 2018). Les élèves ont également fait part de la fréquence à laquelle ils étaient exposés aux PR, mesurée à l'aide de questions relatives à différentes pratiques, telles que : "Lorsque quelqu'un se conduit mal, mes professeurs interrogent les élèves sur leurs versions des faits ", ou « Mes professeurs tiennent compte des idées et opinions de leurs élèves dans la prise de décision » (Gregory, 2018). Les analyses statistiques ont montré que lorsque les élèves font part d'une plus forte exposition aux PR, ils démontrent également des compétences sociales et émotionnelles plus élevées dans les domaines de la conscience de soi et de la régulation émotionnelle/comportementale (Gaines, 2018).

Les preuves corrélationnelles des avantages de ce programme ont été corroborées par la réaction des élèves aux cercles de développement communautaire. Nous avons demandé à des élèves de répondre à une courte enquête, de cinq items, juste après avoir participé à un cercle. La majorité des 124 répondants a indiqué que l'expérience du cercle était positive. Les élèves apprécient les cercles. La plupart les considèrent comme (a) pertinents, (b) bénéfiques pour leur apprentissage et (c) utiles pour développer des relations positives. Plus précisément, $94 \%$ des répondants estimaient que les adultes respectaient leurs idées, $92 \%$ estimaient que les sujets abordés dans le cercle étaient pertinents d'un point de vue personnel, $89 \%$ estimaient avoir appris quelque chose durant le cercle et $78 \%$ estimaient que le cercle aidait à établir des relations positives. Lors d'un entretien, un élève a déclaré :

Quand on participe à des cercles, c'est comme si on exprimait nos pensées et ce qu'on ressent, ce qu'on a envie de dire... On a un bâton de parole, qui aide les élèves à se rappeler que «Hého, quelqu'un est en train de parler, alors il faut se taire. » Ça nous aide... à penser plus et à analyser les questions.

Selon un autre élève : "Les cercles rassemblent des gens pour qu'ils s'expriment sur ce qu'ils ressentent en classe et sur ce qu'ils voudraient que les autres changent ». Une autre élève a expliqué comment, lorsque des personnes partagent quelque chose de triste au sein du cercle, ils peuvent s'identifier les uns aux autres :

"On essaie de réconforter la personne qui se sent mal. Après, on est sur la même longueur d'onde et on comprend ce qu'ils ont vécu».

\section{SYNTHÈSE ET IMPLICATIONS POUR DES PR DANS LES ÉTABLISSEMENTS SCOLAIRES}

À plusieurs reprises au cours de la mise en œuvre du projet, l'adhésion des enseignants s'est écornée du fait d'un phénomène de lassitude lié à la multiplication des initiatives et d'un certain épuisement professionnel/personnel. En outre, comme dans la plupart des établissements, les membres du personnel avaient des convictions divergentes s'agissant du degré de priorité à accorder au développement des relations 
et à la résolution de problèmes par rapport à un objectif unique consistant à délivrer un contenu académique aux élèves. Ils avaient également des croyances culturelles différentes en matière de discipline et quant à savoir si les enseignants devaient adopter une posture autoritaire vis-à-vis des élèves. Cela dit, la capacité des adultes à intégrer les PR à leurs interactions quotidiennes s'est accrue au fil des années du projet. Une infrastructure a en outre été mise en place pour pouvoir bâtir une école plus restaurative. Mais les PR ne sont pas un programme statique et il n'y a pas de fin prédéterminée à ce travail. La durabilité et l'approfondissement des bases posées nécessiteront de la persistance et de la cohérence.

Cette étude de cas offre des leçons pour la diffusion généralisée des PR. Les cercles, énoncés affectifs, processus équitables, conversations et conférences restauratives sont souvent considérés comme des attributs distinctifs des PR. Mais l'efficacité de ces pratiques dépend néanmoins de la capacité des adultes à être vulnérables et à prendre les risques émotionnels nécessaires pour s'engager les uns auprès des autres, auprès des élèves et des familles. Être un « adulte restaurateur » requiert une intelligence émotionnelle, une réflexion personnelle, ainsi que la capacité et la volonté de tisser des relations bienveillantes et de partager le pouvoir avec les élèves, les familles et les collègues (Evans et Vaandering, 2016). Les établissements qui entreprennent des initiatives de PR doivent être attentifs à développer des systèmes, des procédures et des opportunités pour des interactions entre adultes qui favorisent, façonnent et soutiennent cette attente en termes de nécessaire vulnérabilité.

Une autre implication est que de simples ateliers annuels ne peuvent suffire à pérenniser les $\mathrm{PR}$. Les capacités des adultes doivent être régulièrement consolidées par une formation et un coaching à l'échelle du district, s'agissant notamment de la tenue des cercles, de la gestion de classe, des techniques de désescalade et des conférences restauratives. Mais cette formation doit aller au-delà du transfert de compétences spécifiques : comme le montre cette étude de cas, les districts doivent également aider les établissements à entreprendre un changement organisationnel. Les chefs d'établissement ont besoin d'aide pour faire évoluer les systèmes adultes afin de se réorganiser autour des PR, mais également pour mettre en place un suivi continu des progrès. Une réflexion continue, fondée sur des cercles tout au long de l'année, peut aider à résoudre les problèmes d'adaptation qui se poseront à mesure que les membres du personnel éducatif expérimenteront de la démotivation, la réactivation des mentalités punitives, l'émoussement de leur adhésion, ainsi que pléthore d'autres défis qui ne manqueront pas de surgir à mesure qu'ils mettent au point les systèmes et les politiques de restauration. 


\section{RÉFÉRENCES BIBLIOGRAPHIQUES}

AMSTUTZ L. et MULLET J.H. (2005). The little book of restorative discipline for schools. Intercourse, PA : Good Books.

BERKOWITZ K. (2019). "The art of integrating school climate initiatives ». Dans M. THORBORNE, N. RIESTENBERG et G. MCCLUSKEY (coord.), Getting more out of Restorative Practices in schools, Londres : Jessica Kinglsey Publishers.

BOYES-WATSON C. et PRANIS K. (2015). Circle Forward. St. Paul, Minnesota : Living Justice Press.

EVANS K. et VAANDERING D. (2016). The Little Book of Restorative Justice in Education: Fostering Responsibility, Healing, and Hope in Schools. New York : Good Books.

GAINES E. (2018). Restorative practices and student well-being in urban schools. Mémoire non publié, Rutgers University, New Brunswick, New Jersey.

GREGORY A. (2018). RP-Assess: Quality, Quantity, and Equity in Restorative Practices Implementation, v. 2. Recueil de mesures non publié, Rutgers University, New Brunswick, New Jersey.

OWENS J. et MCLANAHAN S. (2018). " Unpacking the drivers of racial disparities in school suspension and expulsion ». Working Papers wp18-04-ff, Princeton University, Woodrow Wilson School of Public and International Affairs, Center for Research on Child Wellbeing.

THORBORNE M., RIESTENBERG N. et MCCLUSKEY G. (2019). Getting more out of Restorative Practices in schools. Londres : Jessica Kinglsey Publishers.

SABOL T.J. et PIANTA R.C. (2012). "Recent trends in research on teacher-child relationships », Attachment \& Human Development, n 14, p. 213-231.

WACHTEL T. (2016). Defining restorative. Bethlehem, Pennsylvania: International Institute for Restorative Practices. [en ligne] [https://bit.ly/2Msl7tb] 
